\title{
Diagnostic significance of serum IncRNA HOTAIR and its predictive value for the development of chronic complications in patients with type 2 diabetes mellitus
}

Huiyun Wang ${ }^{*} \mathbb{D}$, Yu Xia and Yanxia Zhang

\begin{abstract}
Background: Type 2 diabetes mellitus (T2DM) affects the social economy and quality of life, and has become a major threat to human health. This observation aimed to study the possibility of serum HOTAIR as a diagnostic index in patients with T2DM and to explore the prognostic potential of HOTAIR in the development of T2DM.

Methods: The expression of HOTAIR in serum of 96 patients with T2DM and 82 healthy controls was detected by the qRT-PCR technique. The related biochemical indexes of all participants were determined, such as total cholesterol (TC) and fasting blood glucose (FBG). The value of serum HOTAIR in the diagnosis of T2DM in the two groups was analyzed by the ROC curve. Moreover, the prognostic value of HOTAIR on T2DM was examined by the K-M curve and COX multivariate analysis.

Results: The results of the qRT-PCR analysis showed that the serum level of HOTAIR in patients with T2DM was significantly higher than that in healthy controls. ROC analysis showed that HOTAIR in serum was a diagnostic factor of T2DM. Further multivariate analysis showed that HOTAIR could be an independent biomarker in the prediction of chronic complications for T2DM patients, such as diabetic retinopathy and diabetic nephropathy.

Conclusions: We found the augment of HOTAIR expression was a character of T2DM. The high expression of serum HOTAIR was a potential non-invasive diagnostic marker and independent prognostic factor in patients with T2DM.
\end{abstract}

Keywords: diagnosis, prognosis, HOTAIR, T2DM

\section{Background}

Type 2 diabetes mellitus (T2DM) is a common metabolic disorder in the clinic, characterized by high blood glucose and complex pathogenesis, including insulin-resistant $\beta$ cell failure $[1,2]$. The etiopathogenesis of T2DM mainly involves insulin resistance and insulin secretion deficiency [3]. In addition, genetic, environmental, and glycolipid dysfunction also play an important role in

*Correspondence: manyun43@163.com

Department of Health Comprehensive Geriatrics, Yidu Central Hospital of Weifang, No. 4138, Linglongshan Road, Weifang 262500, Shandong, China the occurrence and development of diabetes, which are the main risk factors for vascular lesions $[4,5]$. The vast majority of diabetic complications, if not treated in time, will not only reduce the quality of life of patients but may be life-threatening $[6,7]$. Early prediction and effective treatment of diabetic complications are currently the focus of research [8]. With the development of molecular biology, it is necessary to explore the pathogenesis of diabetes on the molecular level and find the related prediction factors.

Long non-coding RNA (lncRNA) is a class of RNA longer than 200 nucleotides [9]. A variety of lncRNAs original author(s) and the source, provide a link to the Creative Commons licence, and indicate if changes were made. The images or other third party material in this article are included in the article's Creative Commons licence, unless indicated otherwise in a credit line to the material. If material is not included in the article's Creative Commons licence and your intended use is not permitted by statutory regulation or exceeds the permitted use, you will need to obtain permission directly from the copyright holder. To view a copy of this licence, visit http://creativecommons.org/licenses/by/4.0/. The Creative Commons Public Domain Dedication waiver (http://creativeco mmons.org/publicdomain/zero/1.0/) applies to the data made available in this article, unless otherwise stated in a credit line to the data. 
participate in the occurrence and development of T2DM, which was reflected by more and more shreds of evidence. A study finds that IncRNA ENST00000588707.1 is related to glucose metabolism and serves as a feasible biomarker in the field of T2DM [10]. A next-generation sequencing about T2DM discovers that the expression of MALAT1 and MIAT is raised and these abnormal levels have associations with insulin resistance [11]. LncRNA NONRATT021972 is at an increased level in patients with T2DM and its interference may release neuropathic pain and inflammation [12].

In recent years, the investigation has found that HOTAIR is abnormally expressed in several disorders involving T2DM and acts as a satisfactory predictor. The human lncRNA HOTAIR (HOX transcript antisense intergenic RNA) is a 2364 bp lncRNA transcribed from a 6449 bp gene [13]. HOTAIR locates on chr.12q13.13 and consists of six exons [14]. The half-life of HOTAIR has cell-specific variations [15]. The HOTAIR transcript's half-life is approximately $4 \mathrm{~h}$ in HeLa cells [16]. Shaker et al. [17] provide the expression of HOTAIR is raised in diabetic retinopathy patients and it may distinguish diabetic retinopathy from nondiabetic retinopathy. Besides, a report manifests that hyperglycemia contributes to the enhanced HOTAIR expression in HRECs [15]. In diabetic cardiomyopathy, HOTAIR participates in the development of Akt phosphorylation and viability of cardiomyocytes [18]. Additionally, in an experiment about the function of HOTAIR on mice treated with high-fat food, the expression of HOTAIR is significantly elevated, which documents a close correlation between HOTAIR and T2DM [19]. Nevertheless, the clinical significance of HOTAIR in T2DM is not clear.

Hence, this study included T2DM patients and healthy people to detect the change of HOTAIR expression. Moreover, the diagnostic and prognostic values of HOTAIR were identified in our investigation.

\section{Materials and methods \\ Patients and sample collection}

Ninety six newly detected T2DM patients in Yidu Central Hospital of Weifang were selected and divided into the T2DM group. A total of 82 healthy subjects who received physical examinations in the same period were selected and set as the healthy control group. The inclusion criteria of the T2DM group were in accordance with the criteria for diagnosis and classification of diabetes recommended by the World Health Organization in 1999, namely fasting venous plasma glucose $\geq 7.0 \mathrm{mmol} / \mathrm{L}$ [20]. All participants had not received any antidiabetic drugs or insulin treatment before. Patients with other types of diabetes, severe cardiovascular diseases, and malignant tumors were excluded. The elbow venous blood of the two groups was collected in the morning and centrifuged with $1500 \mathrm{r} / \mathrm{min}$ at room temperature for $5 \mathrm{~min}$. The serum samples were collected and stored at $-80{ }^{\circ} \mathrm{C}$. No blood was presented in the serum samples. This study was approved by the Ethics committee of Yidu Central Hospital of Weifang.

All participants were followed up for 5 years after being included in this study. Some chronic complications, including microvascular complications and macrovascular complications, were recorded. Microvascular complications included diabetic nephropathy, diabetic neuropathy, and diabetic retinopathy; macrovascular complications included cerebrovascular disease, cardiovascular diseases, and so on. The follow-up was completed if the endpoint event occurred or a 5-year assessment was conducted.

\section{Detection of HOTAIR expression in T2DM patients}

The total RNA of serum was extracted by the TRIzol method according to the instructions of the TRIzol LS kit. In short, $1 \mathrm{ml}$ TRIzol was mixed with per $200 \mu \mathrm{l}$ serum sample for 5 minutes, and then $200 \mu \mathrm{l}$ chloroform was added to the previous mixture. The supernate was obtained after being centrifuged and isopropanol was added to precipitate RNA. After that, RNA was washed with $75 \%$ ethanol and solubilized with RNase-free water. The OD260/280 value was detected by NanoDrop ${ }^{\text {TM }}$ spec- $^{-}$ trophotometer and only samples with OD260/280 ratio of 1.8-2.0 could be used for cDNA synthesis. Then, the concentration of total RNA was measured with Qubit 3.0. The $5 \times$ gDNA digester Mix in the reverse transcription kit (Yeason, China) was used to remove the residual genomic DNA. A total of $1 \mu \mathrm{g}$ extracted total RNA was reverse transcribed according to the instructions of the reverse transcription kit (Yeason, China, 11121ES60) to synthesize cDNA. PCR reaction was carried out according to the instructions of the TB Green ${ }^{\circledR}$ Premix Ex Taq ${ }^{\mathrm{TM}}$ II (Takara, Japan, RR820A). Namely, $10 \mu \mathrm{l}$ TB Green Premix Ex Taq II, 0.4 $\mu$ l ROX Reference Dye II (50X), 0.8 $\mu \mathrm{l}$ Forward Primer $(10 \mu \mathrm{M}), 0.8 \mu \mathrm{l}$ Reverse Primer $(10$ $\mu \mathrm{M}), 6 \mu \mathrm{l}$ RNase-free water, and $2 \mu \mathrm{l}$ cDNA were mixed on ice. The primers were as follows: HOTAIR forward, 5'-GCAGTGGAATGGAACGGATT-3', HOTAIR reverse, 5'-CGTGGCATTTCTGGTCTTGTA-3'; $\beta$-actin forward, 5'-CCACCATGTACCCAGGCATT-3', $\beta$-actin reverse, 5'-CGGACTCATCGTACTCCTGC-3'. The expression level of HOTAIR in each group of samples was calculated using the 2-DeltaDeltaCt method. $\beta$-actin was used as the internal control [21, 22].

\section{Statistical analysis}

GraphPad Prism and SPSS software were used for statistical analysis. The measurement data of normal 
distribution were expressed by mean \pm standard deviation $(\mathrm{x} \pm \mathrm{s})$. The Kolmogorov-Smirnov test was used to detect whether data conformed to normal distribution. Continuous variables between the two groups were compared by the independent samples $t$ test. Non-parametric data were compared with the Mann-Whitney U test, and categorical data were compared with the chi-square test. Clinical significance of HOTAIR was elucidated by ROC curve and K-M curve. COX multivariate analysis was used to verify whether HOTAIR was an independent indicator in T2DM. $P<0.05$ is statistically significant.

The estimation of sample size was based on preliminary data regarding a study power of $80 \%$ (alpha $=0.05$, beta $=0.2)$ and an effect size of $20 \%$. The sample size required for each group was calculated as 70 and the total sample size required was 140 .

\section{Results}

Baseline clinicopathological features of all participants There were 44 males and 38 females in the control cohort with an average age of $51.07 \pm 6.38$ years, and 51 males and 45 females in T2DM group with a mean age of $50.95 \pm 6.45$. There were no obvious differences in gender, age, and BMI between healthy people and the T2DM group $(P>0.05$, Table 1$)$. The alternation of LDL, TC, TG, and hypertension in the T2DM group was not statistically significant $(P>0.05$, Table 1$)$. The concentration of HDL was lessened in the T2DM patients $(P<0.01$, Table 1). The levels of FBG in the T2DM group were significantly higher than those in the healthy control group

Table 1 Clinical data of the study population

\begin{tabular}{lllr}
\hline Variables & \multicolumn{2}{l}{ All subjects $(\mathbf{n}=\mathbf{1 7 8})$} & P value \\
\cline { 2 - 3 } & $\begin{array}{l}\text { Healthy } \\
\text { individuals } \\
(\mathbf{n = 8 2})\end{array}$ & $\begin{array}{l}\text { T2DM } \\
\text { patients } \\
(\mathbf{n = 9 6 )}\end{array}$ & \\
\hline Gender (male/female) & $44 / 38$ & $51 / 45$ & 0.943 \\
Age (years) & $51.07 \pm 6.38$ & $50.95 \pm 6.45$ & 0.824 \\
BMI (kg/m $\left.{ }^{2}\right)$ & $23.91 \pm 2.09$ & $24.26 \pm 2.05$ & 0.489 \\
HDL (mmol/L) & $1.36 \pm 0.15$ & $1.31 \pm 0.36$ & $<0.01$ \\
LDL (mmol/L) & $2.42 \pm 0.37$ & $2.43 \pm 0.48$ & 0.671 \\
TC (mmol/L) & $4.59 \pm 1.18$ & $4.84 \pm 0.96$ & 0.122 \\
TG (mmol/L) & $1.07 \pm 0.35$ & $1.05 \pm 0.35$ & 0.736 \\
FBG (mmol/L) & $5.04 \pm 0.58$ & $7.77 \pm 0.44$ & $<0.001$ \\
HbA1c (\%) & $4.66 \pm 1.01$ & $6.59 \pm 1.53$ & $<0.001$ \\
Hypertension (yes/no) & $38 / 44$ & $46 / 50$ & 0.834 \\
\hline
\end{tabular}

The data of age, BMI, HDL, LDL, TG, FBG were analyzed by Mann-Whitney U-test. The differences in $\mathrm{TC}$ and $\mathrm{HbA} 1 \mathrm{c}$ were analyzed by the $t$ test method. The chisquare test was used to calculate the differences in gender and hypertension Data are expressed as $\mathrm{n}$ or mean \pm standard deviation

$B M I$ body mass index; $H D L$ high-density lipoprotein; $L D L$ low density lipoprotein; $T C$ total cholesterol; $T G$ triglycerides; FBG fasting blood glucose
$(P<0.001$, Table 1$)$. Besides, the HbA1c content was elevated in the T2DM group compared with the control group $(\mathrm{P}<0.001$, Table 1$)$.

\section{HOTAIR expression in T2DM}

The expression of HOTAIR in T2DM patients and healthy individuals was identified. As demonstrated in Fig. 1, the expression of HOTAIR was raised in the T2DM patients in comparison with control individuals, insisting T2DM might contribute to the enhancement of HOTAIR expression $(P<0.001)$. The expression of HOTAIR in T2DM patients was 1.509 times that of healthy individuals.

\section{Diagnostic performance of HOTAIR}

Considering the alternation of HOTAIR expression in T2DM patients, the diagnostic possibility of HOTAIR was examined by the ROC curve. The AUC of HOTAIR was 0.896 (standard error, $\mathrm{SE}=0.024$ ), and the percentage of sensitivity and specificity was $86.50 \%$ and $84.14 \%$ respectively at the cut-off value of 1.216 (Fig. 2). This finding substantiated HOTAIR could discriminate T2DM patients from control individuals.

\section{Prognosis of HOTAIR in T2DM}

Based on the average expression of HOTAIR in T2DM patients, all patients were divided into the low HOTAIR expression group and high HOTAIR expression group. Fifty patients were included in the high expression of

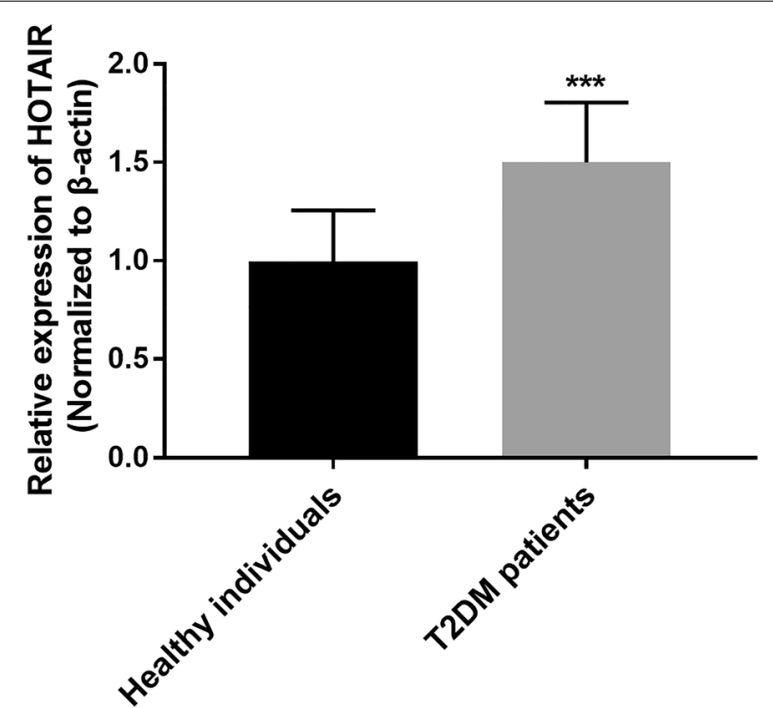

Fig. 1 Elevated expression of HOTAIR was found in 96 patients with T2DM compared with 82 healthy individuals. The method of calculating relative HOTAIR expression was the Livak and Schmittgen method, and the t test was used to compare the differences. ${ }^{* * *} P<0.001$ 


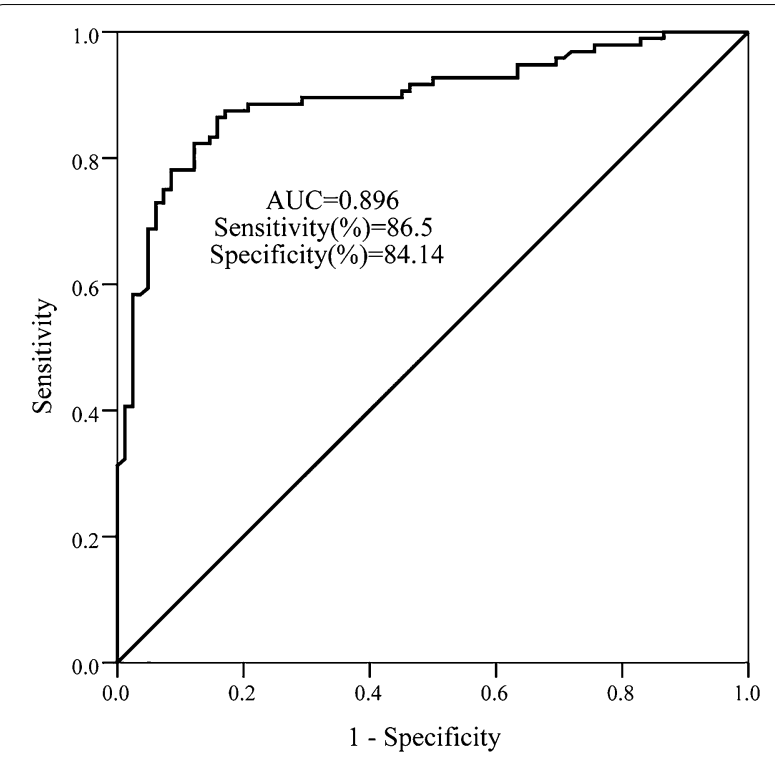

Fig. 2 The diagnostic value of HOTAIR for T2DM patients. A total of 96 T2DM patients and 82 healthy controls were included and a ROC curve was applied to analyze the diagnostic value

HOTAIR group and others were composed of the low expression group. The follow-up revealed that 35 patients had T2DM complications, including 23 patients with microvascular complications, 16 patients with trace urine protein, and 4 patients with retinopathy. The analysis based on the chronic vascular diseases showed that individuals with high HOTAIR expression were more likely to develop chronic complications $(P=0.016$, Fig. 3$)$.

When the healthy control population was used as the dichotomous variable, the results of multivariate regression analysis showed that HOTAIR was an independent risk factor for T2DM complications $(\mathrm{HR}=3.577,95 \% \mathrm{CI}$ 1.349-9.487, $P=0.010$, Table 2). Further, age, sex, BMI, HDL, LDL, TC, TG, and hypertension were used for correction $(P>0.05)$. FBG was an independent risk factor for T2DM complications $(\mathrm{HR}=2.160,95 \%$ CI $1.017-4.586$, $P=0.045$, Table 2). The level of HbA1c was an independent biomarker of T2DM $(\mathrm{HR}=2.484,95 \%$ CI $1.076-$ 5.737, $P=0.033$, Table 2).

\section{Discussion}

T2DM is a common type of diabetes, which basically has a high incidence in the elderly population [23]. T2DM is caused by environmental influence, genetic factors, or metabolic dysfunction [24]. T2DM mainly leads to insulin dysfunction and endocrine function disorders, which will also lead to adipocyte lesions in patients [25]. The high morbidity and mortality of T2DM seriously affected socio-economic condition, life quality, and human health [26]. Chronic vascular complications are the leading

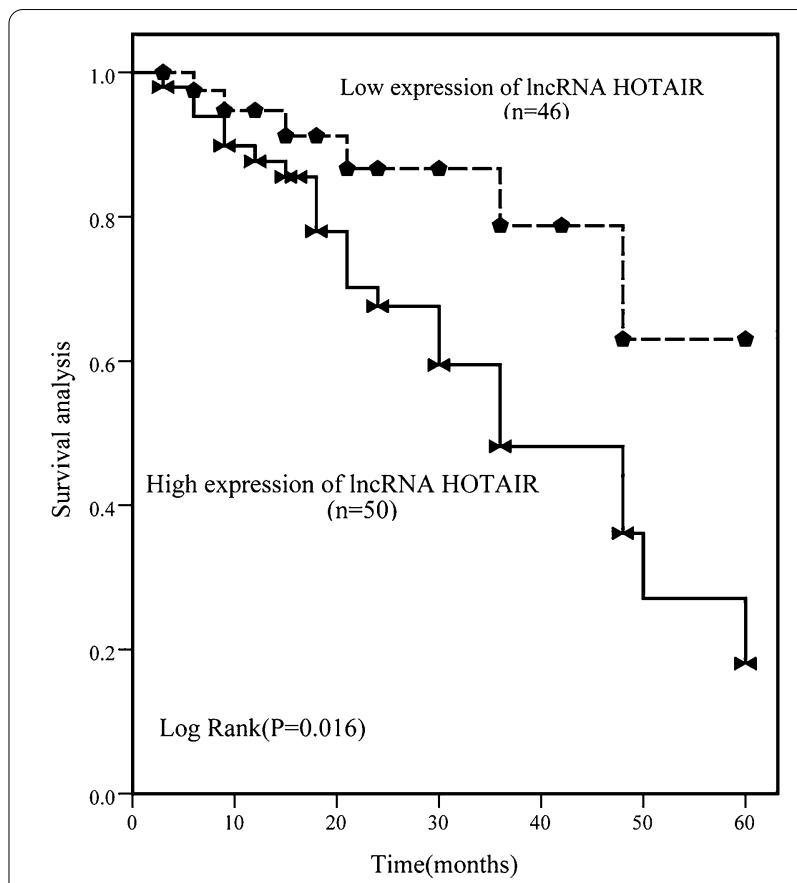

Fig. 3 Diagnostic significance of HOTAIR in T2DM complications was analyzed by K-M curve. There were 46 patients in the low HOTAIR expression group and 50 patients in the high HOTAIR expression group

causes of disability and death in patients with diabetes [27]. Most T2DM patients died of macroangiopathy, and microangiopathy is also the main cause of blindness and renal failure in T2DM [28]. Therefore, chronic vascular complications of diabetes have received more attention.

Table 2 Multivariate Cox analysis of clinical characteristics in relation to overall survival

\begin{tabular}{llll}
\hline Factors & \multicolumn{3}{l}{ Multivariate analysis } \\
\cline { 2 - 4 } & HR & $\mathbf{9 5} \% \mathbf{C l}$ & $\boldsymbol{P}$ \\
\hline LncRNA HOTAIR & 3.577 & $1.349-9.487$ & 0.010 \\
Age & 0.763 & $0.350-1.663$ & 0.497 \\
Gender (male/female) & 0.815 & $0.374-1.772$ & 0.605 \\
BMl (kg/m²) & 0.742 & $0.325-1.695$ & 0.479 \\
HDL (mmol/L) & 0.492 & $0.229-1.055$ & 0.069 \\
LDL (mmol/L) & 1.168 & $0.551-2.473$ & 0.685 \\
TC (mmol/L) & 1.479 & $0.665-3.285$ & 0.337 \\
TG (mmol/L) & 0.632 & $0.299-1.334$ & 0.229 \\
FBG (mmol/L) & 2.160 & $1.017-4.586$ & 0.045 \\
HbA1c (\%) & 2.484 & $1.076-5.737$ & 0.033 \\
Hypertension & 0.707 & $0.338-1.480$ & 0.357 \\
\hline
\end{tabular}

$B M I$ body mass index; HDLhigh-density lipoprotein; LDLlow density lipoprotein; TC total cholesterol; TGtriglycerides; FBG fasting blood glucose 
However, the etiology and pathogenesis of T2DM have not yet been fully elucidated.

A large number of recent studies have shown that lncRNAs participate in the occurrence and development of diabetes and play key regulatory roles. In an experiment, lncRNA MALAT1 is at a raised level in mice with insulin resistance and may be a target of T2DM therapy [29]. MEG3 is elevated in patients with T2DM and its levels are in direct proportion to the concentration of glucose, underlying a novel perspective of pathogenesis of T2DM [21]. Research articles on the significance of HOTAIR have been widely published in the literature [30]. In a report about the function of HOTAIR, HOTAIR facilitates the progress of glucose uptake in the LPS-triggered macrophages via modulating upstream genes of glucose metabolism [31]. Another study unveils that the highly expressed HOTAIR can regulate glucose levels by promoting insulin transcription-related genes, which provides the expression of HOTAIR that may be closely relative to glucose metabolism [32]. Besides, in pancreatic adenocarcinoma, HOTAIR is highly expressed in tumor tissues and can promote energy metabolism of cancer cells by enhancing glucose uptake, lactate production, and ATP synthesis [33]. However, research on HOTAIR in the field of the pathogenesis of T2DM is scarce and its expression in peripheral blood of T2DM patients has not been elucidated yet. In this investigation, the expression of HOTAIR was elevated in T2DM patients, providing that HOTAIR might be involved in the development of T2DM. A report published in 2018 also finds that the expression of HOTAIR is elevated in T2DM patients, which further verified our results [11].

The clinical values of IncRNAs on T2DM have been discussed by several researchers. In a pooled research, lncRNAs are likely to be predictive indicators for T2DM patients after analyzing 7 previous publications [34, 35]. In particular, little is known about the significance of HOTAIR in the prognosis and diagnosis of T2DM. The diagnosis of HOTAIR for T2DM patients was proved in this study, which was derived from the finding that HOTAIR might distinguish T2DM patients from healthy persons with high specificity and sensitivity. LncRNAs have also been approved as prognostic markers for many diabetic complications. In research about diabetic retinopathy, the expression of HOTAIR is raised in patients and used as a novel biomarker in diabetic retinopathy [17]. Besides, the AUC of 0.878 reasons that serum HOTAIR may distinguish diabetic cardiomyopathy patients from healthy individuals [36]. In the current study, the prognostic value of HOTAIR on the diabetes-relative complications was detected by the K-M curve. This analysis indicated that abundantly expressed HOTAIR might be an indicator to predict the occurrence of chronic complications. Moreover, further analysis provided that HOTAIR might be an independent biomarker in the prognosis of T2DM. These findings provided a viewpoint that HOTAIR might associate with the pathogenesis of T2DM and can be used as a significant biomarker. However, some limitations existed in this study, including a small sample size, possible selection bias of patients, and insufficient clinical data. The absence of data on smoking and uric acid from all individuals, which might limit this investigation.

Collectively, this study validated T2DM contributed to the increased HOTAIR levels. The abundance of HOTAIR indicated an increased probability of suffering T2DM. High expression of HOTAIR might be a prognostic indicator because it could lead to a worse outcome of chronic complications. Besides, HOTAIR and FBG were independent biomarkers of T2DM respectively.

\section{Abbreviations}

T2DM: Type 2 diabetes mellitus; BMI: Body mass index; HDL: High-density lipoprotein; LDL: Low density lipoprotein; TC: Total cholesterol; TG: Triglycerides; FBG: Fasting blood glucose.

\section{Acknowledgements}

Not applicable.

\section{Authors' contributions}

HW and YX participated in the design of this study, and they both performed the statistical analysis. HW and YZ carried out the study and collected important background information. HW drafted the manuscript. All authors read and approved the final manuscript.

\section{Funding}

Not applicable.

\section{Availability of data and materials}

The datasets used and/or analyzed during the current study are available from the corresponding author on reasonable request.

\section{Declarations}

Ethics approval and consent to participate

This study was approved by the Ethics committee of Yidu Central Hospital of Weifang. All patients signed a written informed consent form.

Consent for publication

Patients signed informed consent regarding publishing their data.

Competing interests

The authors declare that they have no competing interests.

Received: 9 May 2021 Accepted: 2 September 2021

Published online: 08 September 2021

\section{References}

1. Huang X, Tong Y, Qi CX, Xu YT, Dan HD, Shen Y. Disrupted topological organization of human brain connectome in diabetic retinopathy patients. Neuropsychiatr Dis Treat. 2019;15:2487-502.

2. Horvath A, Leber B, Feldbacher N, Tripolt N, Rainer F, Blesl A, et al. Effects of a multispecies synbiotic on glucose metabolism, lipid marker, gut microbiome composition, gut permeability, and quality of life in 
diabesity: a randomized, double-blind, placebo-controlled pilot study. Eur J Nutr. 2020;59(7):2969-83.

3. Gao Y, Zheng T, Ran X, Ren Y, Chen T, Zhong L, et al. Vitamin D and incidence of prediabetes or type 2 diabetes: a four-year follow-up community-based study. Dis Markers. 2018;2018:1926308.

4. Raza ST, Abbas S, Siddiqi Z, Mahdi F. Association between ACE (rs4646994), FABP2 (rs 1799883), MTHFR (rs1801133), FTO (rs9939609) genes polymorphism and type 2 diabetes with dyslipidemia. Int J Mol Cell Med. 2017;6(2):121-30.

5. Wang J, Zhao D, Ding CZ, Guo F, Wu LN, Huang FJ, et al. MicroRNA-194: a novel regulator of glucagon-like peptide-1 synthesis in intestinal $\mathrm{L}$ cells. Cell Death Dis. 2021;12(1):113.

6. Gouda M, Matsukawa M, lijima H. Associations between eating habits and glycemic control and obesity in Japanese workers with type 2 diabetes mellitus. Diabetes Metab Syndr Obes Targets Ther. 2018;1 1:647-58.

7. Chen Z, Zhang C, Fan G. Interrelationship between interpersonal interaction intensity and health self-efficacy in people with diabetes or prediabetes on online diabetes social platforms: an in-depth survey in China. Int J Environ Res Public Health. 2020. https://doi.org/10.3390/ijerp h17155375.

8. Menini S, lacobini C, Vitale M, Pugliese G. The inflammasome in chronic complications of diabetes and related metabolic disorders. Cells. 2020. https://doi.org/10.3390/cells9081812.

9. Balıı Okcanoğlu T, Kayabaşı Ç, Gündüz C. Effect of CCT137690 on long non-coding RNA expression profiles in MCF-7 and MDA-MB-231 cell lines. Bosn J Basic Med Sci. 2020;20(1):56-62.

10. Ma Q, Wang L, Yang Y, Su Y, Wang T, Hou Q, et al. Association between IncRNA and GCKR gene in type 2 diabetes mellitus. Clin Chim Acta. 2020;501:66-71.

11. Sathishkumar C, Prabu P, Mohan V, Balasubramanyam M. Linking a role of IncRNAs (long non-coding RNAs) with insulin resistance, accelerated senescence, and inflammation in patients with type 2 diabetes. Human Genom. 2018;12(1):41

12. Yu W, Zhao GQ, Cao RJ, Zhu ZH, Li K. LncRNA NONRATT021972 was associated with neuropathic pain scoring in patients with type 2 diabetes. Behav Neurol. 2017;2017:2941297.

13. Zhang J, Chen K, Tang Y, Luan X, Zheng X, Lu X, et al. LncRNA-HOTAIR activates autophagy and promotes the imatinib resistance of gastrointestinal stromal tumor cells through a mechanism involving the miR-130a/ATG2B pathway. Cell Death Dis. 2021;12(4):367.

14. Cantile M, Di Bonito M, Cerrone M, Collina F, De Laurentiis M, Botti G. Long non-coding RNA HOTAIR in breast cancer therapy. Cancers. 2020. https://doi.org/10.3390/cancers12051197.

15. Biswas S, Feng B, Chen S, Liu J, Aref-Eshghi E, Gonder J, et al. The long non-coding RNA HOTAIR is a critical epigenetic mediator of angiogenesis in diabetic retinopathy. Investig Ophthalmol Vis Sci. 2021;62(3):20.

16. Bhan A, Mandal SS. LncRNA HOTAIR: a master regulator of chromatin dynamics and cancer. Biochim Biophys Acta. 2015;1856(1):151-64.

17. Shaker OG, Abdelaleem OO, Mahmoud RH, Abdelghaffar NK, Ahmed $\mathrm{TI}$, Said OM, et al. Diagnostic and prognostic role of serum miR-20b, miR-17-3p, HOTAIR, and MALAT1 in diabetic retinopathy. IUBMB Life. 2019;71(3):310-20.

18. Qi K, Zhong J. LncRNA HOTAIR improves diabetic cardiomyopathy by increasing viability of cardiomyocytes through activation of the PI3K/Akt pathway. Experimental Ther Med. 2018;16(6):4817-23.

19. Li M, Guo Y, Wang XJ, Duan BH, Li L. HOTAIR participates in hepatic insulin resistance via regulating SIRT1. Eur Rev Med Pharmacol Sci. 2018;22(22):7883-90.
20. Alvarez-Silva C, Kashani A, Hansen TH, Pinna NK, Anjana RM, Dutta A, et al. Trans-ethnic gut microbiota signatures of type 2 diabetes in Denmark and India. Genome Med. 2021;13(1):37.

21. Chang WW, Zhang L, Yao XM, Chen Y, Zhu LJ, Fang ZM, et al. Upregulation of long non-coding RNA MEG3 in type 2 diabetes mellitus complicated with vascular disease: a case-control study. Molecular Cell Biochem. 2020;473(1-2):93-9.

22. Wang L, Su N, Zhang Y, Wang G. Clinical significance of serum IncRNA cancer susceptibility candidate 2 (CASC2) for chronic renal failure in patients with type 2 diabetes. Medical Sci Monit Int Med J Exp Clin Res. 2018;24:6079-84

23. Fittipaldi E, Andrade AD, Santos ACO, Campos S, Fernandes J, Catanho M. Depressive symptoms are associated with high levels of serum low-density lipoprotein cholesterol in older adults with type 2 diabetes mellitus. Arquivos Bras Cardiol. 2020;115(3):462-7.

24. Lee D, Han Y, Kwon EY, Choi MS. d-allulose ameliorates metabolic dysfunction in C57BL/KsJ-db/db mice. Molecules. 2020. https://doi.org/10. 3390/molecules25163656.

25. Bhansali S, Bhansali A, Dutta P, Walia R, Dhawan V. Metformin upregulates mitophagy in patients with T2DM: a randomized placebo-controlled study. J Cell Mol Med. 2020;24(5):2832-46.

26. Chen J, Zheng CX, Jin Y, Hu CH. Mesenchymal stromal cell-mediated immune regulation: a promising remedy in the therapy of type 2 diabetes mellitus. Stem Cells. 2021. https://doi.org/10.1002/stem.3357.

27. Peng $X$, Wang $X$, Fan $M$, Zhao J, Lin L, Liu J. Plasma levels of von Willebrand factor in type 2 diabetes patients with and without cardiovascular diseases: a meta-analysis. Diab Metab Res Rev. 2020;36(1):e3193.

28. Liu X, Men P, Wang Y, Zhai S, Liu G. Impact of dipeptidyl peptidase-4 inhibitors on serum adiponectin: a meta-analysis. Lipids Health Dis. 2016;15(1):204

29. Liu SX, Zheng F, Xie KL, Xie MR, Jiang LJ, Cai Y. Exercise reduces insulin resistance in type 2 diabetes mellitus via mediating the IncRNA MALAT1/ microRNA-382-3p/resistin axis. Mol Ther Nucleic Acids. 2019;18:34-44.

30. Cantile M, Di Bonito M, De Tracey BM, Botti G. Functional interaction among IncRNA HOTAIR and microRNAs in cancer and other human diseases. Cancers. 2021. https://doi.org/10.3390/cancers13030570.

31. Obaid M, Udden SMN, Alluri P, Mandal SS. LncRNA HOTAIR regulates glucose transporter Glut1 expression and glucose uptake in macrophages during inflammation. Sci Rep. 2021;11(1):232

32. Zhu HP. Silence of HOTAIR inhibits insulin secretion and proliferation in pancreatic $\beta$ cells. Eur Rev Med Pharmacol Sci. 2020;24(2):784-92.

33. Ma Y, Hu M, Zhou L, Ling S, Li Y, Kong B, et al. Long non-coding RNA HOTAIR promotes cancer cell energy metabolism in pancreatic adenocarcinoma by upregulating hexokinase-2. Oncol Lett. 2019;18(3):2212-9.

34. Zhang W, Zheng J, Hu X, Chen L. Dysregulated expression of long noncoding RNAs serves as diagnostic biomarkers of type 2 diabetes mellitus. Endocrine. 2019:65(3):494-503.

35. Saeidi L, Ghaedi H, Sadatamini M, Vahabpour R, Rahimipour A, Shanaki M, et al. Long non-coding RNA LY86-AS1 and HCG27_201 expression in type 2 diabetes mellitus. Mol Biol Rep. 2018;45(6):2601-8.

36. Abdulle LE, Hao JL, Pant OP, Liu XF, Zhou DD, Gao Y, et al. MALAT1 as a Diagnostic and Therapeutic Target in Diabetes-Related Complications: A Promising Long-Noncoding RNA. Int J Med Sci. 2019:16(4):548-55.

\section{Publisher's Note}

Springer Nature remains neutral with regard to jurisdictional claims in published maps and institutional affiliations. 